\title{
A NOVEL DERIVATIVE ELLIPSOMETRIC METHOD FOR THE STUDY OF THE GROWTH OF THIN FILMS: TITANIUM
}

\author{
J. M. M. DE: NIJS AND A. VAN SII.FHOUT \\ Twente Lnitersity. Faculty of Applied Phisics. P.O. Box 217.7500 AE Enschede 'The Netherlands, \\ (Received June 30. 1988; revised December 14. 1988; accepted January 31. 1989)
}

The growth of a titanium film at room temperature from an evaporation source on a silicon substrate covered by its native oxide layer is continuously monitored with an ellipsometer at three wavelengths. The momentary positions and the derivatives of the trajectories thus obtained in the $(A, \Psi)$ plane can be used for uniquely determining the momentary thickness and the momentary dielectric constants of the layer at each of the wavelengths. The optical properties of the titanium, which reflect the film structure and defect rate, strongly depend upon the growth conditions; the top region of a film approximately $40 \mathrm{~nm}$ thick appears to contain more voids and lattice defects than the region near the substrate.

1. INTRODUCTION

During the last decade, spectroscopic ellipsometry has become a technique which has been shown to provide very detailed information on a system under study' ${ }^{\prime}$. A prerequisite is that the dielectric functions of the constituent components are accurately known. Concerning thin film applications, the dielectric function should be obtained from a well defined thin film. The problem in this case is that a simple ellipsometric measurement yields only two quantities at each wavelength, a $\Delta$ value and a $\Psi$ value, which is not sufficient to calculate the dielectric constant as well as the layer thickness ${ }^{5}$. Growing the film so thick that it becomes completely opaque up to the ellipsometric sensitivity is not always advised. In particular, high-melting-point metals deposited at room temperature by vapor deposition techniques are expected to yield films of a poor quality, compared with sputter-deposited films, because the metal atoms from an evaporation source have a substantially lower kinetic energy than the sputtered atoms. The larger kinetic energy of the latter permits these atoms to move to the preferential atomic locations, whereas in the case of vapor deposited high-melting-point metals, the atoms almost instantaneously freeze in at the location where they condense. Therefore it is expected that these layers contain voids and defects whereas the amount of voids and defects may vary with the film thickness ${ }^{6.7}$. 
The problem of obtaining the dielectric function is as well as the later thickness d from ellipsometric measurements can be countered in several wats. All these methods hate in common that the vary one of the system parameters. providing one with the extra information reyuired to solve the problem. (One can vary the ambient ${ }^{x}$. the layer thick ness" or alternatively. one catn vats the angle of incidence $(i) \mid 11,1$. Regarding thin films grown under ultrahigh vacuum (l'HW conditions, it will be most obvious to bary the layer thick ness An altermative bery effective method. which can also be used in this case, is the technigue proposed by Arwin and Aspnes? They have shown that in those cases where the substrate dielectric function has a very characteristic structure. one can oblain the later thick ness by eliminating any substrate related structure in the culculated psededodielectric function of the film. Here we present an alternative technique. a variant of the thickness variation technique. which we have applied for the determination of the dielectric function of a titanium film grown under I IHV conditions. The film growth is followed at three wavelengths during the deposition process. In this way we obtain complete trajectories of the film growth in the $(1, \Psi)$ plane. With these trajectories we also have derivative d.1. $\mathrm{d} \psi$ for each of the wavelengths for every data point at our disposal, which is a quantity independent of measured 1 and $\psi$ values. Subsequently, we can calculate the momentary dielectric constants and latyer thickness. The reliability of this technique is improved by coupling of the corresponding data points of the three wavelengths. One of the advantages of the method is that not only is the layer thick ness yielded during the deposition. but also the homogeneity of the film is checked from the instantaneous dielectric constants. Furthermore it is better than the method of Arwin and Aspnes in that it cat be used in those cases where the substrate dielectric function lacks any pronounced features. This can be of great importance if one studies reactive layers. wich require a specific substrate. It should be emphasized that this method is less successful the closer in value the dielectric constants of the top layer and the substrate.

Our new method is illustrated for the growth of a titanium layer. Dielectric functions of titanium can be found in the literature. but the agreement among the reported functions is very poor ${ }^{1.3}{ }^{15}$. These differences are due to a number of reasons, mainly sample preparation and measuring technique: however. surface roughness. oxide layers, anisotropy, grain size and the degree of porosity or amorphousness strongly influence the magnitude of the dielectric function. Apart from this structural sensitivity of the dielectric function, we have to deal with the crystal orientation of titanium. Titanium has an h.c.p. unit cell. which, in general. requires a dielectric tensor function for a proper description.

This uncertainty of the dielectric function of metals obstructs an accurate evaluation of the ellipsometric spectra, and one is advised to obtain the dielectric function from a titanium film which is grown under equivalent conditions as the layers to be studied ${ }^{13}$. Therefore we have grown a thin titanium film at room temperature from a resistively heated titanium wire on a silicon substrate which is covered by a native oxide layer. Such a film does not react with the $\mathrm{SiO}_{2}$ layer underneath ${ }^{16}$ and is expected to be polycrystalline with no preferential grain orientation $^{6.7 .1^{7} .18}$. Therefore, we presume that a dielectric tensor function is not required, but that a dielectric scalar function suffices to describe the dielectric 
response of the titanium film. Bulk dielectric functions for $\mathrm{c}-\mathrm{Si}$ are obtained from ref. 19.

This paper can be summarized as follows. Firstly, we introduce the measurements and the results obtained. Subsequently we discuss the method of the analysis, and the paper closes with a discussion of the actual results obtained. A comparison is made between the performance of the present method and that of Arwin and Aspnes. In the present case of a titanium film on an $\mathrm{Si} / \mathrm{SiO}_{2}$ system, the performance of the newly presented method will prove to be better in comparison with that of Arwin and Aspnes. Finally we present some linear regression analyses (LRA) of the titanium layers obtained upon a prolonged growth up to about $40 \mathrm{~nm}$. This shows that the optical properties of the top region have changed dramatically.

\section{EXPERIMENTAL DETAILS}

Measurements were performed with a fully automated rotating analyser ellipsometer $(E=2.0-4.5 \mathrm{eV})^{20.21}$ equipped with mercury and xenon lamps. The titanium was evaporated from a resistively heated wire inside a UHV system. During the film growth the pressure did not rise above $4 \times 10^{-9} \mathrm{mbar}$. Prior to the film growth we performed a spectroscopic ellipsometric measurement, from which the thickness of the oxide layer was calculated. During the deposition process we successively measured the ellipsometric parameters $\Delta$ and $\Psi$ at three wavelengths (the 365,435 and $545 \mathrm{~nm}$ lines of the mercury lamp) at equidistant time points $t_{i}$. Initially, the values of $\Delta$ were close to $180^{\circ}$, obstructing accurate measurements unless a compensator was used ${ }^{22,23}$. For this reason the initial film growth was omitted and the measurements were commenced at a film thickness of about $8 \mathrm{~nm}$ without the use of a compensator. In general, and particularly when windows are required, one is advised to use a two-zone measurement eliminating all first-order errors except the window errors ${ }^{23.24}$. However, because we desire a time-dependent measurement with as many data points as possible at three wavelengths, we performed "semi" two-zone measurements: the first and final measurements were real two-zone measurements, while the intermediate data-points were single-zone measurements. Subsequently, the zone differences of the first and final measurement were used to correct the single-zone results. These measures allowed us to perform the ellipsometric measurements at the three wavelengths with a precision better than $0.01^{\circ}$ for $\Delta$ and $0.005^{\circ}$ for $\Psi$ within $30 \mathrm{~s}$. The final accuracy was restricted by the birefringence of the windows, which caused errors in $A$ of $1^{\prime \prime}-2^{\circ}$ at most; $\Psi$ was not affected. After termination of the deposition process one final two-zone spectroscopic ellipsometric scan was performed. In Fig. 1 we have plotted the data points thus obtained in the $(\Delta, \Psi)$ plane. From every trajectory the $i$ th data point is related to the ith data point of each of the other trajectories. It should be emphasized that the presented data analyses were not performed in real time but after we had finished the measurements.

After the spectroscopic ellipsometric scan, we continued the titanium deposition for another $40 \mathrm{~min}$. Subsequently we characterized this layer by a second ellipsometric scan. 


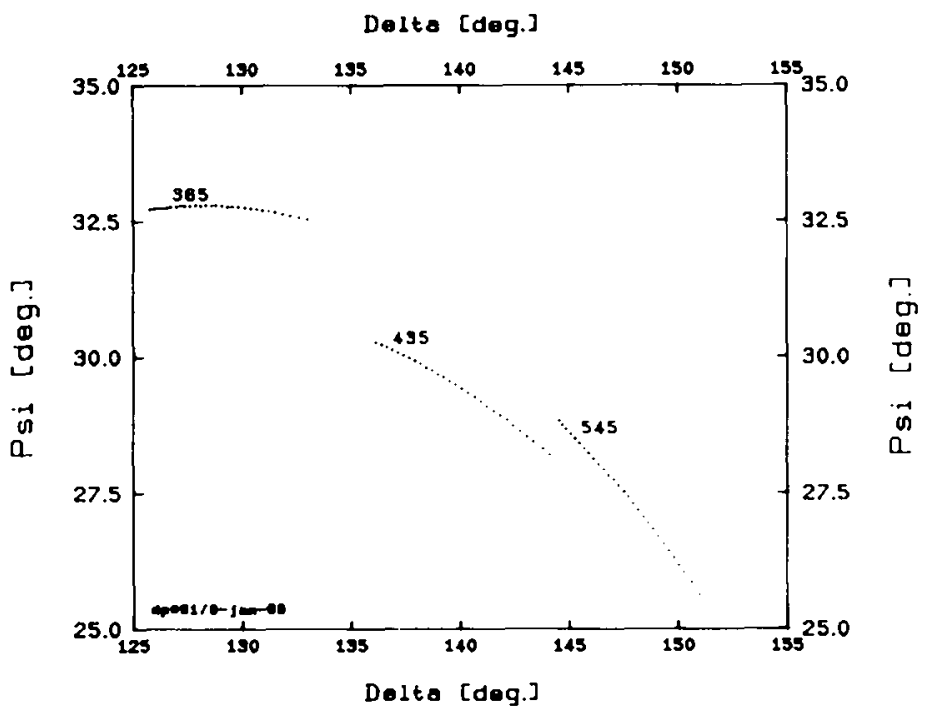

Fig. 1. The deposition of titanium on an Si SiO), substrate has been followed ellipsometrically al three wavelengths. The figure depicts the trajectories drawn in the $\left(1, \Psi^{\prime}\right.$ ) plane.

\section{DATA ANAI.YSIS}

The data points from the three trajectories can be combined into a set $S$. such that every set element $S_{i}$ contains the values of $A$ and $\psi$ at the three wavelengths at time point $l_{1}$ :

$$
S=\left(.1_{i}^{305}, \Psi_{i}^{305}, .1_{i}^{435}, \Psi_{i}^{435}, 1_{i}^{545}, \Psi_{i}^{545}\right)
$$

Every set element $S_{1}$ now is a momentary characterization of the $\mathrm{Si}: \mathrm{SiO}_{2}: \mathrm{Ti}_{\text {sistem }}$ at time point $t_{i}$. Presently we shall assume that the titanium film grows homogeneous and isotropic, allowing us to use a two-layer model for the interpretation of the data; a c-Si substrate covered by its native oxide layer on top of which the titanium film is growing. Prior to the titanium deposition we determined the thickness of the oxide layer from a spectroscopic ellipsometric scan. It was found to be $2.7 \mathrm{~nm}$.

The characterization from the momentary ellipsometric data of the titanium film requires seven parameters: the real and imaginary parts of the dielectric function at each of the wavelengths $\dot{\varepsilon}_{(j)}\left(\lambda_{\text {) }}\right.$ and the thickness $d_{i}$. The set elements $S_{i} d o$ contain six measured quantities for each time point, which is not sufficient for a unique determination of the unknown parameters. However, because we have the complete trajectories at our disposal, we can calculate the derivatives $\mathrm{d} J /\left.\mathrm{d} \Psi\right|_{i}$ for every data point, which is an extra, independent quantity. Extending the sets $S$ with the derivatives, we obtain $\mathbf{T}$ :

$$
\mathrm{T}=\left\{A_{i}^{365}, \Psi_{i}^{365},\left.\left.\left.\frac{\mathrm{d} A}{\mathrm{~d} \Psi}\right|_{i} ^{365} \cdot A_{i}^{435} \cdot \Psi_{i}^{435} \cdot \frac{\mathrm{d} A}{\mathrm{~d} \Psi}\right|_{i} ^{435} \cdot A_{i}^{545} \cdot \Psi_{i}^{545} \cdot \mathrm{d} A\right|_{i} ^{545}\right\}
$$

In this way we have increased the number of independent measured quantities from 
six to nine creating a mathematically redundant situation for calculating uniquely the seven unknown parameters for every set element $T_{i}$.

Next, we have to calculate the dielectric constants $\tilde{\varepsilon}_{i}$ for each wavelength and the thickness of the titanium film $d_{i}$ from the individual set-elements $T_{i}$, which is achieved from the minimalization of the error function $F_{i}$ :

$$
\begin{aligned}
F_{i}= & \sum_{i}\left\{A_{i}-\Delta\left(\tilde{\varepsilon}(\dot{\lambda}), d_{i}\right)\right\}^{2}+\left\{\Psi_{i}-\Psi\left(\tilde{\varepsilon}_{i}\left(\lambda_{i}\right), d_{i}\right)\right\}^{2} \\
& +g\left\{\left.\frac{\mathrm{d} \Delta}{\mathrm{d} \Psi}\right|_{i}-\frac{\mathrm{d} \Delta}{\mathrm{d} \Psi}\left(\tilde{\varepsilon}_{i}(\dot{\lambda}), d_{i}\right)\right\}^{2} \quad i=365,435,545 \mathrm{~nm}
\end{aligned}
$$

The quantity $\mathrm{d} \Delta / \mathrm{d} \Psi$ has a qualitatively different character from the quantities $\Delta$ and $\Psi$. Problems which eventually follow from this difference are met by the introduction of a weight factor $g$, which has to be adjusted such that the influence of the derivative $\mathrm{d} \Delta / \mathrm{d} \Psi$ becomes comparable in strength compared with the influence of $A$ and $\Psi$. Increasing $g$ augments the emphasis on the derivatives and vice versa. If $g$ is chosen too large, the influence of $\Delta$ and $\psi$ becomes completely overruled, which in fact diminishes the number of independently known quantities from nine to three, resulting in an underdefined problem. However, if $g$ is chosen too small, the influence of the derivative $\mathrm{d} \Delta / \mathrm{d} \Psi$ becomes completely dominated by the quantities $\Delta$ and $\Psi$, also yielding an underdefined system. The minimalization is finally performed by a Levenberg-Marquardt routine which yields the optimized parameters as well as their confidence limits ${ }^{25.26}$. The latter provide a good check as to whether the weight factor has been chosen properly.

Firstly, we studied the influence of the weight factor $g$ on the inversion. In Figs. 2 and 3 we show the layer thicknesses $d_{i}$ and the dielectric constants at $\lambda=435 \mathrm{~nm}$ as calculated from the set elements $T_{i}$. These calculations have been performed for

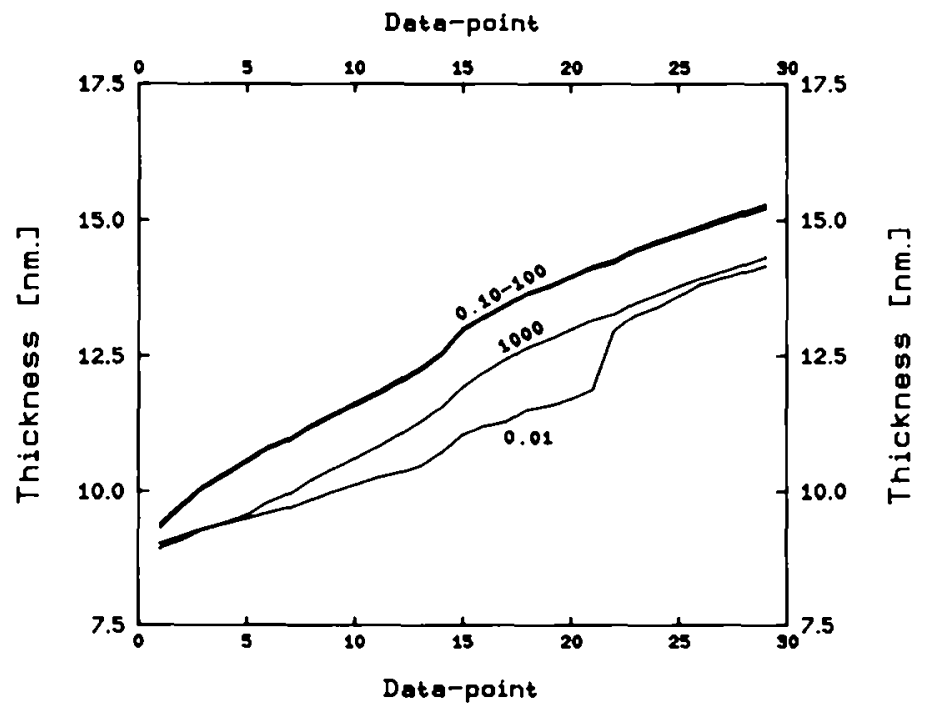

Fig. 2. The thickness of the growing titanium layer as it is obtained by the derivative method. The calculations are performed with different values for the weight factor $g$. 


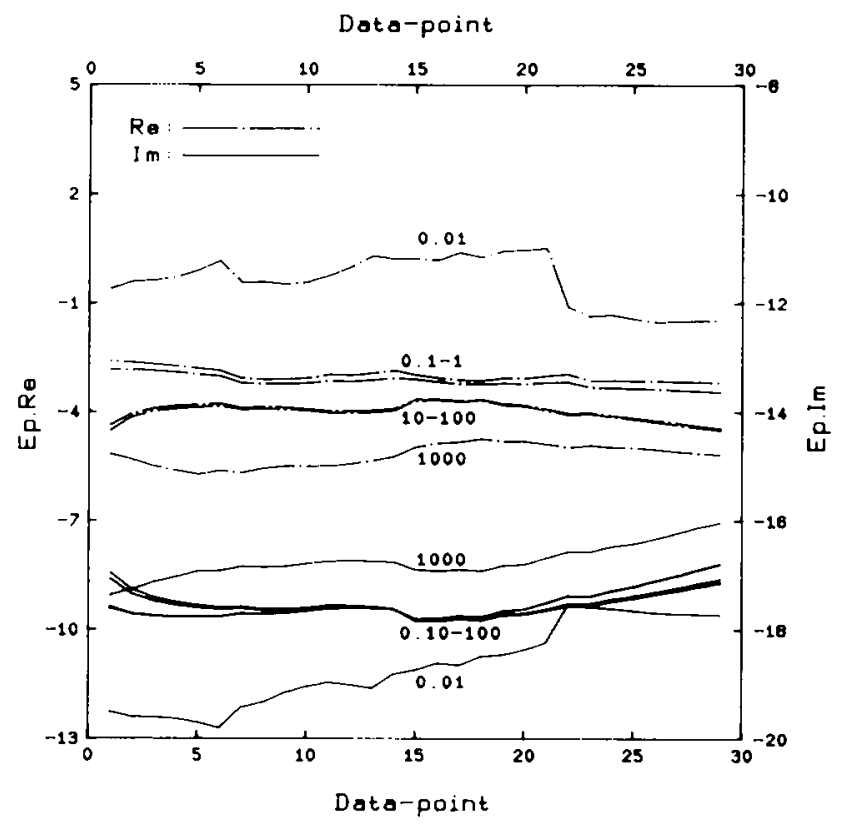

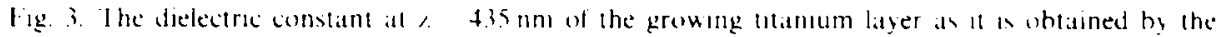
deriative method. The calculations are perlormed with different values for the weight factur 2.

$g=0.01 .0 .10 .1 .0,10,100$ and 1000 . Except for $g=0.01$ or 1000 , all results agree very well. For $g=0.01$ or 1000$)$, it is clearly shown that the influence of either 1 and $\Psi$ or the derivative d $1 \mathrm{~d} \Psi$ becomes suppressed too strongly, leaving a less defined system of equations. Table I contains the limits of confidence for the dielectric constants and the thickness. as calculated for $T_{22}$, which is just an arbitrary but representative set element. confirming that the weight factor $g$ can be safely chosen between $g=0.10$ and $g=100$. This method works well over a large range of $g$. but its performance diminishes abruptly once $g$ is chosen out of the range. For the proceeding calculations we have used $g=10$.

\section{TABI:I: I}

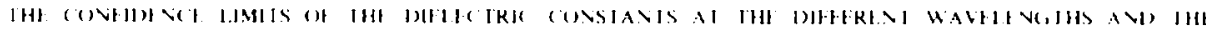

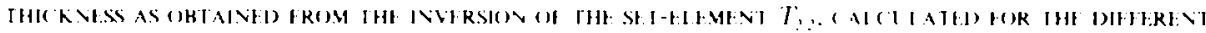

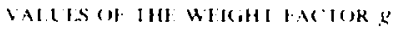

\begin{tabular}{|c|c|c|c|c|c|c|c|}
\hline$g$ & : & & 4.35 & & $54 i$ & & d \\
\hline & $\mathrm{Re}$ & $\mathrm{Im}$ & $\mathrm{Re}_{\mathrm{c}}$ & $\mathrm{Im}$ & $\mathrm{Re}$ & $\operatorname{lm}$ & $(\mathrm{nm})$ \\
\hline $10 x(x)$ & 1).46 & 0.06 & 0.55 & (1) $4 x$ & 1000 & 0.10 & 0.55 \\
\hline $10(x)$ & 01.3 & 0.611 & 0.15 & (1) 14 & 0.25 & 0.14 & 11. 18 \\
\hline 10 & 0.19 & 11.62 & 0.25 & 0.10 & 0.33 & 0.04 & 018 \\
\hline 1 & 0.54 & 11.115 & 0.73 & קבוס & 11.84 & 0.14 & $01 x$ \\
\hline (). 1 & 0.55 & 0.10 & 10.76 & $00 \times 1$ & 0.84 & $11.1 ?$ & $0.1 x$ \\
\hline 0,01 & 240 & 11.56 & 3.10 & 0.16 & 330 & 1.111 & 0.80 \\
\hline
\end{tabular}




\section{RESULTS}

The dielectric constants $\tilde{\varepsilon}_{i}$ for $\dot{\lambda}=365,435$ and $545 \mathrm{~nm}$, as they arise from the inversion of the set elements $T_{i}$, are shown in Fig. 4 , We started this study from the idea that the titanium film would grow homogeneously, which would have resulted in dielectric constants independent of layer thickness. In fact we observe some dependency, mainly for the real parts of the dielectric constants. This can partially be at tributed to the calculations because the largest confidence limits are found for real parts of the dielectric constants for $i=435$ and $545 \mathrm{~nm}$. However, the variations are too large to be attributed solely to the numerical calculations. Statistical errors from the ellipsometric measurements can be ruled out because they are too small; their variances are $\sigma_{1}=0.01$ ' and $\sigma_{\Psi} 0.005$. There remains only one reason: that the layer does not in fact grow homogeneously. However, the variation of the dielectric constants during the titanium deposition is not too worrisome if compared with disagreement among the dielectric constants published in the literature ${ }^{13.14}$. If we study the layer thickness (Fig. 3) we note that all evaluations with $g=0.1$ up to $g=100$ have yielded the same result: that the confidence limit is small $(0.18 \mathrm{~nm})$, pointing to a very accurate determination of the thickness. The final layer thickness was $15.25 \pm 0.18 \mathrm{~nm}$. To check the result, we also applied the method of Arwin and Aspnes $^{12}$. In Fig. 5 we have plotted both real and imaginary parts of the dielectric function of the film as calculated from the spectroscopic ellipsometric scan with different assumptions for the value of the film thickness: $d=14.0,15.0,15.5,16.5 \mathrm{~nm}$ and $d=15.25 \mathrm{~nm}$, the last-mentioned being the result of the Arwin-Aspnes technique. Both dielectric functions which are calculated with the assumption that

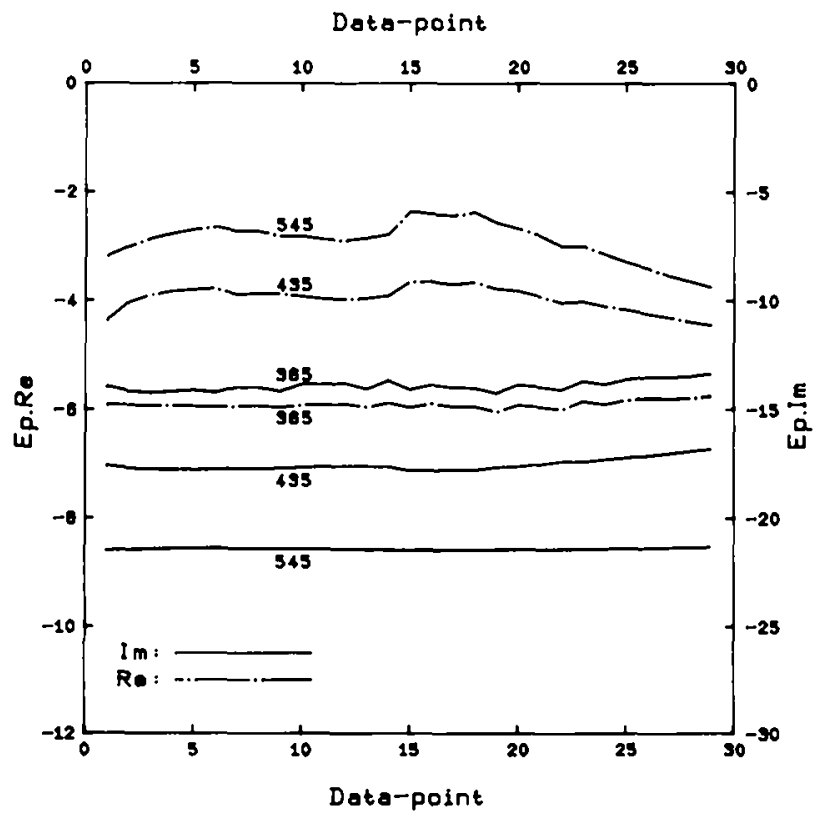

Fig. 4. The dielectric constant at the different wavelengths of the growing titanium layer as it is obtained by the derivative method. The calculations are performed with $g=10$. 


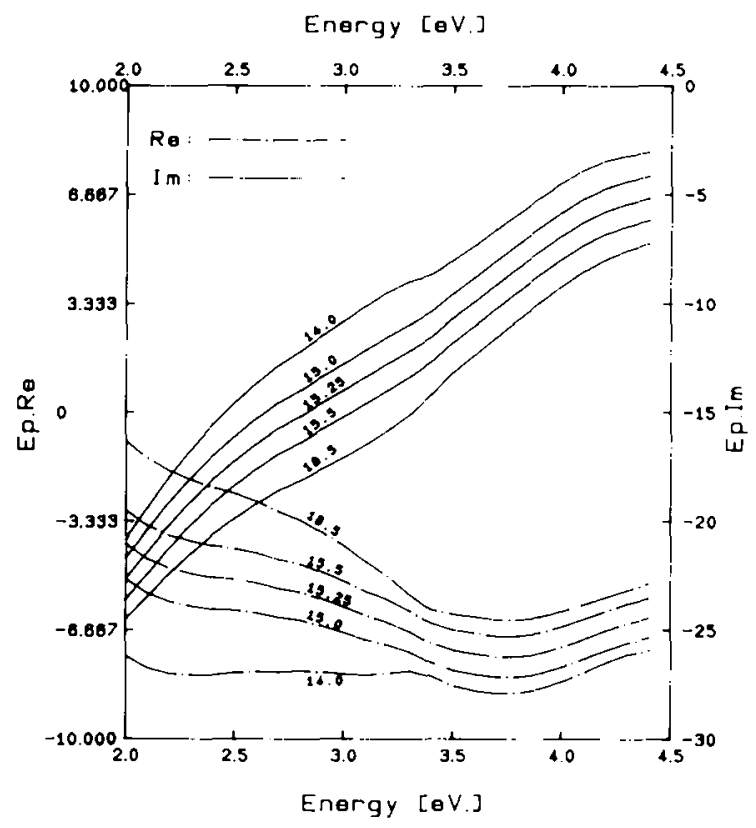

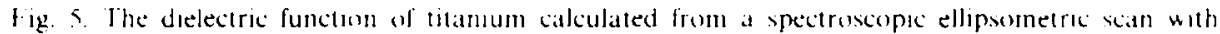
different assumptions on the layer thickness. The commencement of the aboorbing region of the c-Si spectrum $(f=3 . \mathrm{eV})$ is clearly obsersed when the thickness is w rongly chosen

the layer is 14.0 or $16.5 \mathrm{~nm}$ clearly show features to be ascribed to the dielectric function of the substrate. In particular the commencement of the absorbing region of the silicon dielectric function (at $E=3.4 \mathrm{eV}$ ) introduces an artificial kink in the spectra. This kink is minimized, but still noticeable. for all dielectric functions calculated with $d=15.0-15.5 \mathrm{~nm}$ : it shows that the thickness has to be about $15.25 \mathrm{~nm}$, but it is impossible to designate the one showing the least features. The accuracy of the estimation of the thickness shall be $0.5 \mathrm{~nm}$ at most. The fact that the minimization of the structure cannot eliminate the structure completely. is a confirmation of film inhomogeneity.

As already mentioned, the dielectric functions are known for their disagreement. which makes it difficult to compare our results against them. None the less, we have plotted our dielectric function for an evaporated titanium film together with results obtained by Johnson and Christy ${ }^{1 ?}$ in Fig. 6. Johnson and Christy also determined the dielectric function on an evaporated titanium film, which makes their results the most appropriate for a comparison. There is a good qualitative agreement which can be marked by being much better than among the reported dielectric functions in general. The imaginary part is lower than that of Johnson and Christy, which generally is an indication of a film of better quality ${ }^{15}$.

After we had interrupted the deposition process at $d \approx 15.25 \mathrm{~nm}$ and made the spectroscopic ellipsometric scan, we proceeded the film growth for about $40 \mathrm{~min}$. The layer obtained was then characterized by a second spectroscopic ellipsometric scan. This scan was analyzed by means of linear regression analysis (LRA) ${ }^{27} 2^{29}$. We were able to use the presently obtained dielectric function of titanium as the 


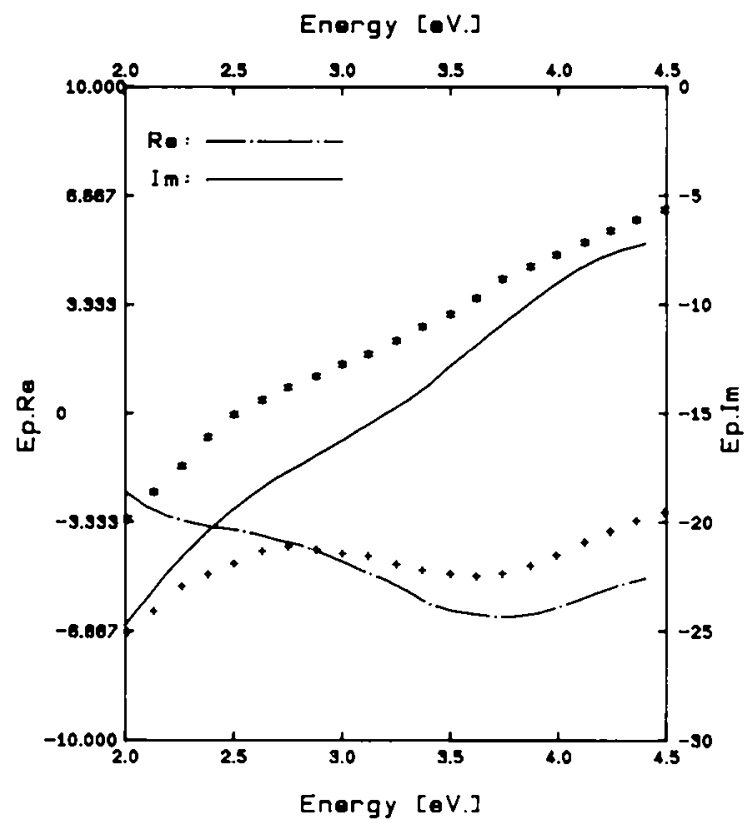

Fig. 6. The presently obtained dielectric function (drawn curves) of titanium and the dielectric function as obtained by Johnson and Christ ${ }^{15}$.

reference dielectric function. If the prolongated growth were to yield a layer of a different optical character, this could be modelled by means of voids ${ }^{15}$; it would be assumed that the layer was not pure metal but contained a certain volume fraction of voids. Subsequently, one could calculate the effective dielectric function of the diluted film from the dielectric functions of the metal and voids $\left(\varepsilon_{\text {voids }}(E)=1\right)$ and the Bruggeman effective medium approximation ${ }^{15.30} 32$. Three different models are compared: a pure layer of titanium on top of the system c-Si/SiO ${ }_{2}$ (model I), a titanium top layer that contains a variable amount of voids (model II) and (III) two titanium top layers, the first containing both metal and voids and the second pure titanium. Detailed examples of such analyses can be found in the literature ${ }^{27}{ }^{29}$. We have optimized the various layer thicknesses and the void fractions by means of a Levenberg-Marquardt routine ${ }^{25}$. The results are listed in Table II.

The first model, a single pure titanium layer, has yielded a very erroneous result; the residue $(\sigma \approx 0.06)$ is quite poor, in particular because we have used the

\section{TABLE II}

IIINEAR REGRESSION A.NALYYSES FOR MODFIS I. II ANI) II!, SHOWING, APAR I FROM THE OPTIMIZFI) PARAMETERS. THF: UNCERTAINTY I.IMITS AND THE RESIDUE:

\begin{tabular}{|c|c|c|c|c|}
\hline Model & $\begin{array}{l}d_{1} \\
(\mathrm{~nm})\end{array}$ & $f_{1}$ & $\begin{array}{l}d_{2} \\
(\mathrm{~nm})\end{array}$ & Residue \\
\hline I & $51 \pm 5$ & & - & 0.06 \\
\hline [I & $40 \pm 0.04$ & $0.871 \pm .001$ & & 0.004 \\
\hline [II & $27 \pm 1.0$ & $0.872 \pm .001$ & $14 \pm 1.5$ & 0.002 \\
\hline
\end{tabular}


previously determined dielectric function of titanium. Apparently we cannot apply this dielectric function without some adaptation. Including a certain number of woids in the titanium layer model Ill drastically improwes the fit: it shows unambiguously that the optical propertics of the layer have changed during the prolonged layer growth. The third model reduces the residue even further lsee Fig. 7). It gives an indication that the initally grown layer has remained the same whereas the subsequently deposited layer contains a latger number of woids. The void-rich top layer is quite thick and its uncertainty limit is about $1 \mathrm{~mm}$. Therefore we can exclude surface roughening as a possible explanation for the large woid fraction in the top later. and we have lo attribute the presence of the roids to a reduction of the layer yuality in the top-region. ie. the prolonged metal deposition has enhanced the growth of defects and possibly micropores.

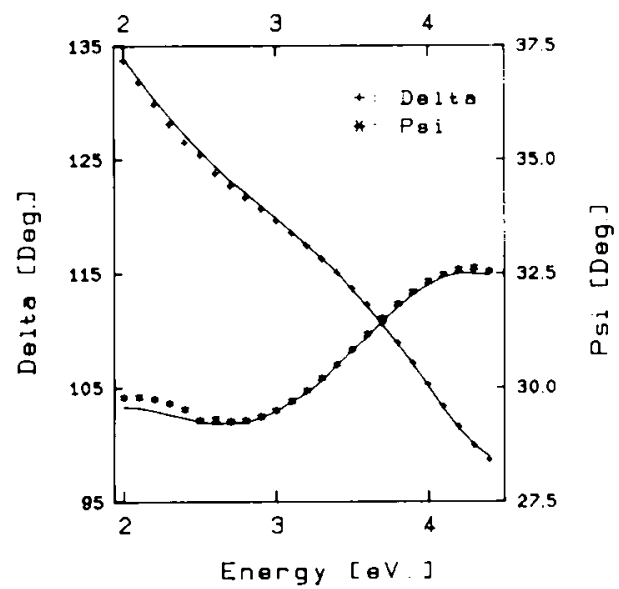

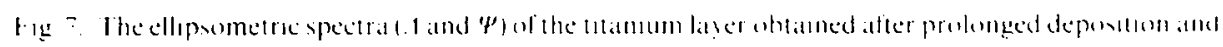

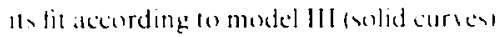

5. I) ISC (15sion

We have shown that the derivative $d .1 \mathrm{~d} \psi$ can be used as a source for supplementary information in the interpretation of ellipsometric data. This method has yielded reliable values for the momentary layer thickness for the present carse of an absorbing layer on a $\mathrm{C}-\mathrm{Si}$ substrate. Combining three wavelengths offers the advantage that the different wavelengths yield complementary information. in particular in the present case where we have used a $c-S i$ substrate whose dielectric function is strongly wavelength dependent. Apart from the thickness. we hate been able to calculate the momentary dielectric constants for the different wavelengths from the trajectories. These momentary dielectric constants offer the possibility of following the homogeneity of the laver grown. Therefore it would be rer! interesting. for the modern thin film and device industry. if one were able to perform the analyses in real time: one would then hate a method to control the quality of a layer during its deposition. an option which maly be realised with the ever-increasing capacity of computers. 
In this work we have studied the growth of an absorbing layer on a c-Si substrate, but non-absorbing or slightly absorbing films constitute another important field for modern thin film technology. For these cases the method presented can be applied as long as the absorbance of the substrate does not become too large. Regarding strongly absorbing substrates, one should realize that the total complex reflection coefficient of the system is dominated by the vacuum/ad-layer reflection, whereas the importance of the reflection of the electromagnetic field at the ad-layer-substrate interface diminishes with increasing absorbance of the substrate; i.e. the complex reflection coefficient does not contain any information on the thickness of the layer but is solely governed by the dielectric properties of the adlayer. This, however, is evident from the confidence limits calculated, which, as said previously, provide a good check on the trustworthiness of the result.

Concerning the titanium films that we have grown, it appears to be difficult to grow a perfectly homogeneous high-melting-point metal film at room temperature by means of evaporation, even under ideal circumstances. Apparently the dielectric properties (which depend on the film structure) are very sensitive to deposition conditions. This probably has to be attributed to a restricted lateral mobility of the titanium atoms after they have condensed on the surface.

\section{RFFFRENCFS}

1 D. E. Aspnes, SPIE Proc . 276 (1981) I88.

2 J. B. Theeten and M. Frman. J. Vac. Sci. Technol. 20(1982) 471.

3 R. W. Collins, B. G. Jacobi, K. M. Jones and Y. S. Tsuo, J. Vac. Sci. Terchnol., A. 4(1986) 153.

4 P. J. Mcmarr. K. Vedam and J. Narayan. J. Appl. Phrs., 59(1986) 694.

5 R. M. A. Azzam and N. M. Bashara, Ellipsometry and Polurized Light. North-Holland, New York. 1977.

6 M. Schneider, A. Rahman and I. K. Schuller, Phis. Re' .. 55(1985)604.

7 K. H. Müller, Surf. Sir.. 184 (1987) 375.

8 F. Lukes, W. H. Knausenberger and K. Vedam. Surf. Sci. $16(1969) 112$.

9 S. S. So and K. Vedam. J. Opt. Soc. Am. 62(1972) 16.

10 G. H. Bu-Abbud and N. M. Bashara. Appl. Opt. 20(1981) 3020.

11 O. Hunderi, Surf. Sci..6J(1976) 515.

12 H. Arwin and D. E. Aspnes, Thin Solid Films. 1/3(1984) 101.

13 J. II. Weaver. C. Krafka, D. W. Lynch and E. E. Koch. Phisics Data. Optical Properties of Metals. Karlsruhe, 1981.

14 E. D. Palik. Handhook of Optical Constants of Solids, Academic Press, New York. 1985.

15 D. E. Aspnes. E. Kinsbron and D. D. Bacon. Phis. Re' B. B. 2/ (1980) 3290.

16 G. J. P. Krooshof, F. H. P. M. Habraken, W. F. van der Weg, L. van den Hove, K. Maex and R. I: de Keersmaecker. J. Appl. Phys.. 63(1988) 5110.

17 P. B. Johnson and R. W. Christy. Phys. Re't. B. 9(1974) 5056.

I8 F. J. van Loenen, A. E. M. J. Fischer \& J. F. van der Veen. Surf. Sci., 155 (1985) 65.

19 D. F. Aspnes and A. A. Studna. Phis. Ret. B. 27 (1983) 985.

20 D. E. Aspnes and A. A. Studna. J. Appl. Opt. 14 (1975) 220.

21 T. Holtslag, Ph.D. Thesis. Twente University, Enschede. 1986.

22 D. E. Aspnes, J. Opt. Soc. Am., 64 (1974)639.

23 J. M. M. de Nijs and A. van Silfhout. J. Opt. Soc. Am. A. 5 (1988) 773.

24 R. M. A. Azzam and N. M. Bashara. J. Opt. Soc. Am. 64(1974)639.

25 D. W. Marquardt. J. Soc. Indust. Appl. Math.. I/ (1963) 431

26 S. Y. Kim and K. Vedam, Appl. Opt., 25 (1986) 2013.

27 R. W. Collins, B. G. Yacobi, K. M. Jones and Y. S. Tsuo, J. Vac. Sit. Technol. A. 4(1986) 153. 


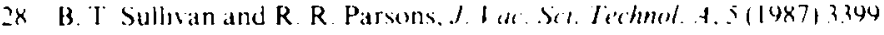

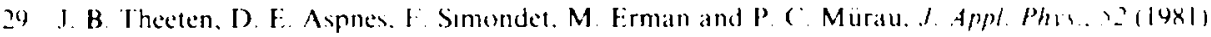
6788

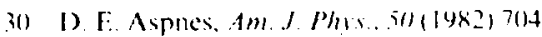

3 I) H. Aspnes. J. B. Thecten and F. Holler. PhW Rer. R. 2011979, 3242

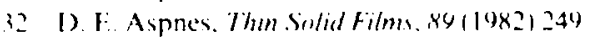

